\title{
Situación pre y post-incendio, de un ecosistema del tipo forestal Roble-Hualo, Región del Maule, Chile
}

\author{
Pre and post-fire situation, of an ecosystem of the Roble-Hualo forest type, Maule \\ region, Chile
}

Jorge Alejandro Rosales-Rodríguez $\bullet$ Edwin Antonio Esquivel-Segura² • Manuel Alejandro Acevedo-Tapia ${ }^{3}$

- Marta González-Ortega ${ }^{3}$ • Eduardo Cartes-Rodríguez ${ }^{3}$

\section{Abstract}

Forest fires are one of the major causes of ecosystem degradation in Chile. The 2016-2017 forest fire season was notable for the affected area, including a forest and scrub, of the Roble-Hualo forest type, of high conservation value. Therefore, this study aimed to evaluate the effect of the fire in both formations, on the structure of the canopy through the use of hemispheric photographs. In addition, the effect of the fire on chemical variables of soil, diversity and abundance of vegetation were quantified. The results indicated a clear degradation of the structure of the canopy of the forest and scrub, increase in the potential radiation on the forest floor, reduction of the foliar area and coverage of the canopies on the ground. Also, some chemical variables such as $\mathrm{pH}$, organic matter (MO\%), sum of bases $\left(\mathrm{S}_{b}\right)$ and available potassium $\left(\mathrm{K}_{\mathrm{d}}\right)$ increased in the scrub soil, but not in the forest floor. Post-fire regeneration was scarce in both coverages, in addition there was a considerable loss of arboreal and shrubby diversity, thus affecting the composition of the vegetation, in the forest and in the scrub.

Key words: Forest fires, forest, hemispheric photographs, soil.

1. Ingeniero independiente; Naranjo, Costa Rica; jrosales050@gmail.com

2. Escuela de Ingeniería Forestal, Tecnológico de Costa Rica; Cartago, Costa Rica; eesquivel@tec.ac.cr 


\section{Resumen}

Los incendios forestales son una de las mayores causas de degradación de ecosistemas en Chile. La temporada de incendios forestales 2016-2017 destacó por la superficie afectada, incluyendo un bosque y matorral, del tipo forestal Roble-Hualo, de alto valor de conservación. Por lo que este estudio tuvo como objetivo evaluar el efecto del incendio en ambas formaciones, sobre la estructura del dosel mediante la utilización de fotografías hemisféricas. Además, se cuantificó el efecto del incendio en variables químicas del suelo, diversidad y abundancia de la vegetación. Los resultados indicaron una clara degradación de la estructura del dosel del bosque y matorral, aumento en la radiación potencial sobre el piso forestal, reducción del área foliar y cobertura de los doseles sobre el suelo. Asimismo, algunas variables químicas como el $\mathrm{pH}$, materia orgánica $(\mathrm{MO} \%)$, suma de bases $\left(\mathrm{S}_{\mathrm{b}}\right)$ y potasio disponible $\left(\mathrm{K}_{\mathrm{d}}\right)$ aumentaron en el suelo del matorral, no así en el suelo del bosque. La regeneración post-incendio fue escaza en ambas coberturas, además se apreció una considerable pérdida de diversidad arbórea y arbustiva, afectando así la composición de la vegetación, en el bosque y en el matorral.

Palabras clave: ncendios forestales, bosques, fotografías hemiesféricas, suelo.

\section{Introducción}

Junto con la transformación de hábitats para urbanización, agricultura, ganadería y silvicultura, los incendios forestales han sido uno de los mayores causantes de perturbación sobre los ecosistemas nativos de Chile [1-3].

Se ha documentado que sólo el 0,1\% de los incendios forestales, registrados en las últimas dos décadas, desde la zona centro de Chile hasta la Patagonia, podrían deberse a causas naturales (figura 1) [1], [3].

En Chile, se registran en promedio 5972 incendios forestales por temporada, los cuales afectan entre 20000 y 85000 ha de vegetación [3]. Más del $70 \%$ de la superficie afectada corresponde a bosques y matorrales nativos, provocando así, grandes pérdidas de biodiversidad y servicios ecosistémicos de valor irreemplazable [3]. Sin embargo, la temporada de incendios forestales 2016-2017, destacó por la superficie afectada, estimada en más de 510000 ha [58].

$\mathrm{Y}$, aun cuando se ha planteado que algunos incendios ocurrieron naturalmente en algunos ecosistemas mediterráneos y templados de Chile, desde antes de la llegada del ser humano, la frecuencia de estos incendios no parece haber sido suficientemente alta como para haber promovido adaptaciones de resistencia o tolerancia, [3], [4] por lo que, prácticamente, no existen ecosistemas naturales dependientes del fuego. Sin escapar de esta realidad se encuentran los bosques y matorrales del tipo forestal Roble-Hualo [3], [5].

Este tipo forestal es endémico de la zona central de Chile, ubicado en la Cordillera de la Costa y en la Cordillera de los Andes, específicamente entre los $35^{\circ}$ y $37^{\circ}$ latitud sur figura 2).

La especie que domina en este tipo forestal corresponde al Nothofagus glauca (Phil.) Krasser (Hualo), sin embargo, se pueden encontrar otras especies, como por ejemplo Persea lingue (Ruiz \& Pav.) Nees (Lingue), Gevuina avellana Molina (Avellano), Nothofagus obliqua (Mirb.) Oerst (Roble) y Nothofagus alessandrii Espinosa (Ruil) [6].

Cabe destacar que, este tipo forestal se encuentra escasamente representado dentro del Sistema Nacional de Áreas Silvestres Protegidas del Estado (SNASPE), aproximadamente sólo el 0,5\% del área ocupada por este ecosistema está protegido [6], [7].

Sumado a la poca representatividad de los ecosistemas, en los sistemas nacionales de áreas silvestres protegidas, los incendios causan graves daños en los bosques y matorrales de este tipo forestal [6-8].

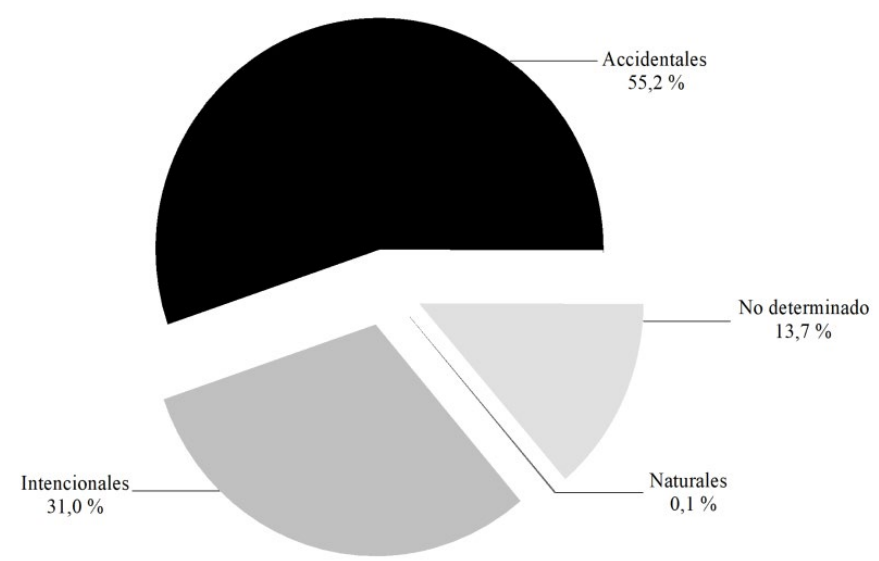

Figura 1. Causas principales de los incendios forestales en Chile, fuente Fernández et al. [3].

Figure 1. Main causes of forest fires in Chile, source Fernández et al [3]. 


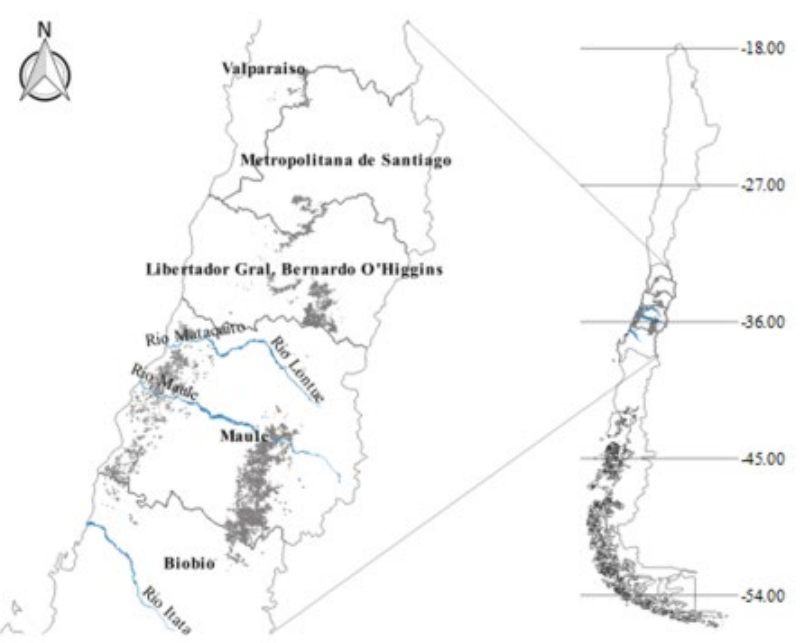

Figura 2. Distribución geográfica del tipo forestal Roble-Hualo, por regiones, fuente elaboración propia a partir de capas del IDE-Chile [59].

Figure 2. Geographic distribution of the Roble-Hualo forest type, by region, source prepared by the IDE-Chile layers [59].

Es así que, como resultado de los incendios forestales y dependiendo de la intensidad y severidad del incendio se puede producir la destrucción parcial o total de el o los doseles presentes en los bosques y matorrales [9].

Además los incendios pueden provocar consecuencias sobre otros componentes del ecosistema, como por ejemplo, aumento de la radiación solar incidente sobre el piso forestal, variables químicas del suelo; abundancia y diversidad de especies arbóreas y arbustivas, entre otros [10].

Comprender los efectos del fuego en los ecosistemas es fundamental, para la gestión de los mismos, en miras a la conservación y aumento del recurso [2]. Al respecto, en este estudio, se evalúan los efectos de uno de los incendios forestales de la temporada 2016-2017, sobre la estructura del dosel, química del suelo, diversidad y abundancia de especies arbóreas y arbustivas de un bosque y matorral del tipo forestal Roble-Hualo, ubicado en la Región del Maule, Chile.

\section{Materiales y métodos}

\section{Área de estudio}

El área de estudio corresponde a un bosque y matorral, de aproximadamente 15 ha, del tipo forestal RobleHualo, ubicado en el predio Quivolgo 4, patrimonio de Forestal Arauco S.A., en la comuna de Constitución, provincia de Talca, región del Maule, Chile (figura 3).

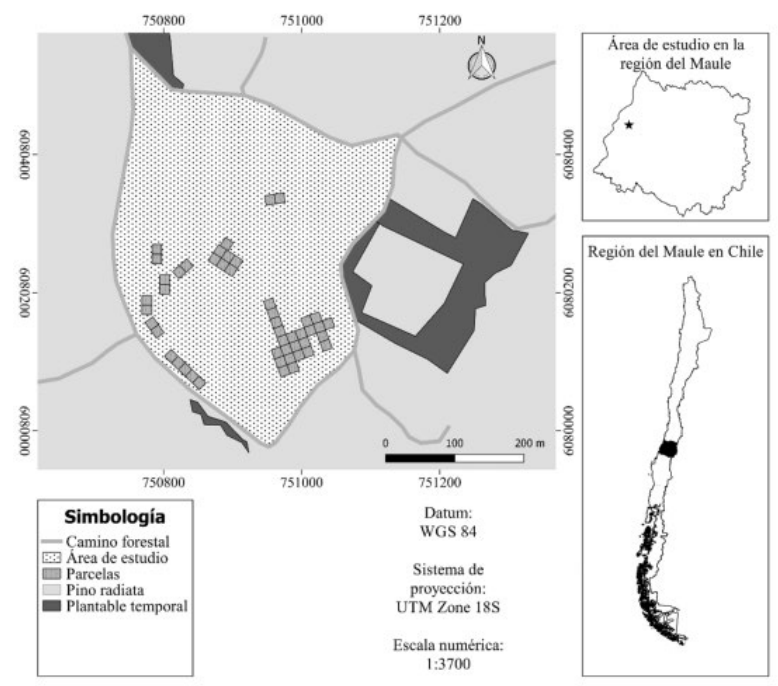

Figura 3. Ubicación geográfica del sitio de estudio, fuente elaboración propia a partir de capas de Albers [60].

Figure 3. Geographical location of the study site, source made by Albers layers [60].

\section{Establecimiento de parcelas en campo}

En la figura 4 se muestra el croquis de las parcelas que se utilizaron para el presente estudio, se reestablecieron las parcelas 26, 27 y 46 en el bosque y 2, 6 y 17 en el matorral, el resto de parcelas que se pueden observar se perdieron a causa del incendio (figura 4). Parcelas de $14 \mathrm{~m} \times 14 \mathrm{~m}$.

\section{Captura y análisis de fotografías hemisféricas para la caracterización de la estructura del dosel}

Para la captura de las fotografías se utilizó una cámara Canon EOS REBEL T5i, con un lente Sunex $185^{\circ}$

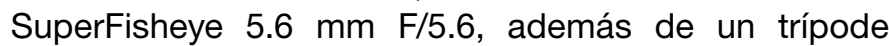
SOLIGOR VT-6006.

En la condición pre-incendio se capturaron 5 fotografías por parcela, mientras que para la condición post-incendio se capturaron 15 fotografías por parcela, aleatoriamente distribuidas dentro de las mismas. Las fotografías para la condición pre-incendio se capturaron el 4 de febrero del 2016 y para la condición post-incendio, el 3 de agosto del 2017.

Para el análisis de las fotografías hemisféricas se utilizaron los softwares HemiView Forest Canopy Image Analysis System 2.1.1@ y Microsoft Office Excel 2016 TM. Mediante la utilización de HemiView 2.1.1@ se calculó el factor global de sitio (GSF), índice de área foliar (LAl) e índice de cobertura de suelo (GndCover).

EI GSF, corresponde a la proporción de radiación global, directa (DSF) más difusa (ISF), bajo un dosel forestal, 


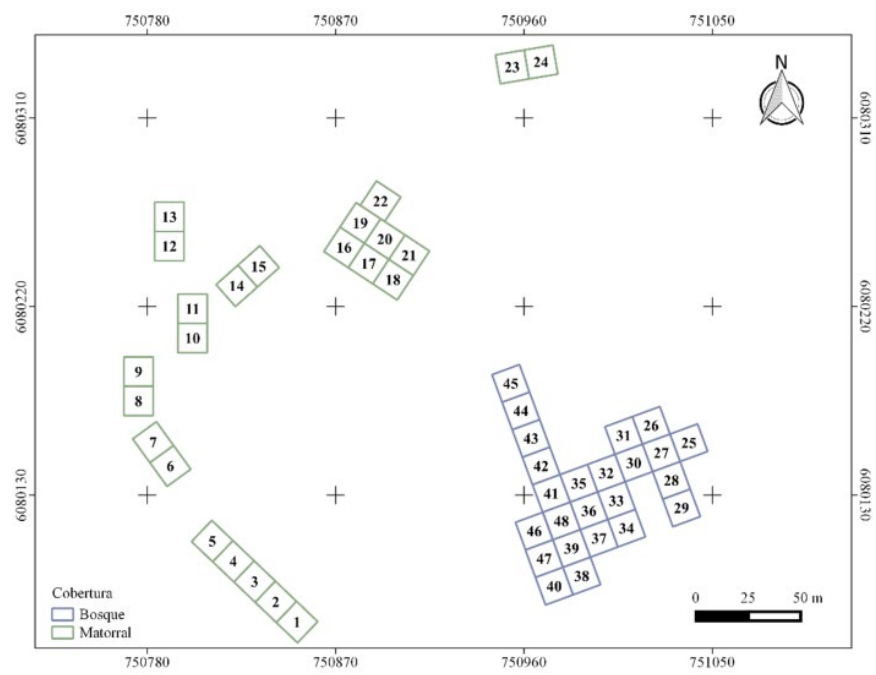

Figura 4. Parcelas de estudio, fuente Acevedo [61].

Figure 4. Study plots, Acevedo source [61].

relativo a campo abierto. El LAl se define como la mitad del área foliar total por unidad de superficie del suelo. Por su parte el GndCover hace referencia a la proporción del área que cubre un dosel en específico con respecto a una unidad de área del suelo [62].

\section{Muestreo de suelos y análisis químico}

En la condición pre-incendio se realizaron dos calicatas, una por cobertura. En la calicata del matorral se colectaron dos muestras, una de 0 a $5 \mathrm{~cm}$ de profundidad, en el primer horizonte, y otra de 5 a $52,5 \mathrm{~cm}$ de profundidad, en el segundo horizonte; en la calicata de la cobertura de bosque se colectó una muestra de 0 a $20 \mathrm{~cm}$ de profundidad, en el primer horizonte.

Para la colecta de las muestras de suelo post-incendio se utilizó un barreno tipo espiral. Se realizó un muestreo compuesto de diez puntos, distribuidos de forma aleatoria dentro de cada parcela, para los primeros 20 $\mathrm{cm}$ de suelo. La colecta de muestras pre-incendio se llevó a cabo el 4 de febrero del 2016, mientras que para la condición post-incendio, el 4 de agosto del 2017.

Se determinó el $\mathrm{pH}$, contenido de materia orgánica (MO\%), nitrógeno disponible ( $\mathrm{Nd})$, suma de bases $(\mathrm{Sb})$, fósforo de Olsen $(\mathrm{Po})$ y potasio disponible $(\mathrm{Kd})$, para los suelos pre y post-incendio y de ambas coberturas. Los análisis se encargaron al laboratorio de suelos de la facultad de agronomía, departamento de suelos y recursos naturales de la Universidad de Concepción, campus Chillán, según los Métodos de Análisis Recomendado para los Suelos de Chile, Serie de Actas
INIA N³4, Revisión 2006 [63] y Métodos de Análisis para Tejidos Vegetales, Serie de Actas INIA N40, 2007 [64].

\section{Caracterización de la abundancia y diversidad de especies arbóreas y arbustivas}

Tanto en la condición pre como post-incendio, y para ambas coberturas, se realizó el conteo e identificación taxonómica, dentro de cada parcela en su totalidad, de individuos de especies arbóreas y arbustivas superiores a $1 \mathrm{~m}$ de altura. Para la condición post-incendio, la colecta de datos se llevó a cabo en octubre del 2017.

\section{Análisis estadísticos}

Para la comparación del GSF, LAl y GndCover, entre coberturas y condiciones se empleó un análisis longitudinal mediante el software estadístico SAS, con tres unidades muestrales por cobertura, y cinco unidades observacionales por unidad muestral en la condición preincendio, y quince unidades observacionales por unidad muestral en la condición post-incendio. El estadístico de discriminación de medias utilizado fue Tukey.

Para el análisis de variables químicas del suelo y abundancia y diversidad de especies arbóreas y arbustivas se procedió a realizar las comparaciones entre condiciones y coberturas por medio de un análisis gráfico.

\section{Resultados y discusión}

\section{Estructura del dosel, para ambas condiciones y coberturas}

En la condición pre-incendio, en las tres variables cuantificadas, GSF, LAl y GndCover, se obtuvieron diferencias estadísticamente significativas entre las coberturas de bosque y matorral, con $\mathrm{P} \leq 0,05$ para el GSF y LAl, y $P \leq 0,01$ para GndCover .

En cuanto al GSF para la cobertura de bosque el valor promedio correspondió a $0,28 \pm 0,02$; por otra parte, para el matorral el valor promedio correspondió a 0,77 $\pm 0,16$ (Figura 6); lo que indica que antes del incendio, la cobertura por la cual podía atravesar mayor cantidad de radiación correspondía al matorral, con un 0,49 $\pm 0,18$ más de radiación potencial total.

Es importante mencionar que en doseles muy cerrados, como es el caso de la cobertura de bosque preincendio, la luz que atraviesa el dosel y llega al suelo está compuesta principalmente por ISF y una fracción 


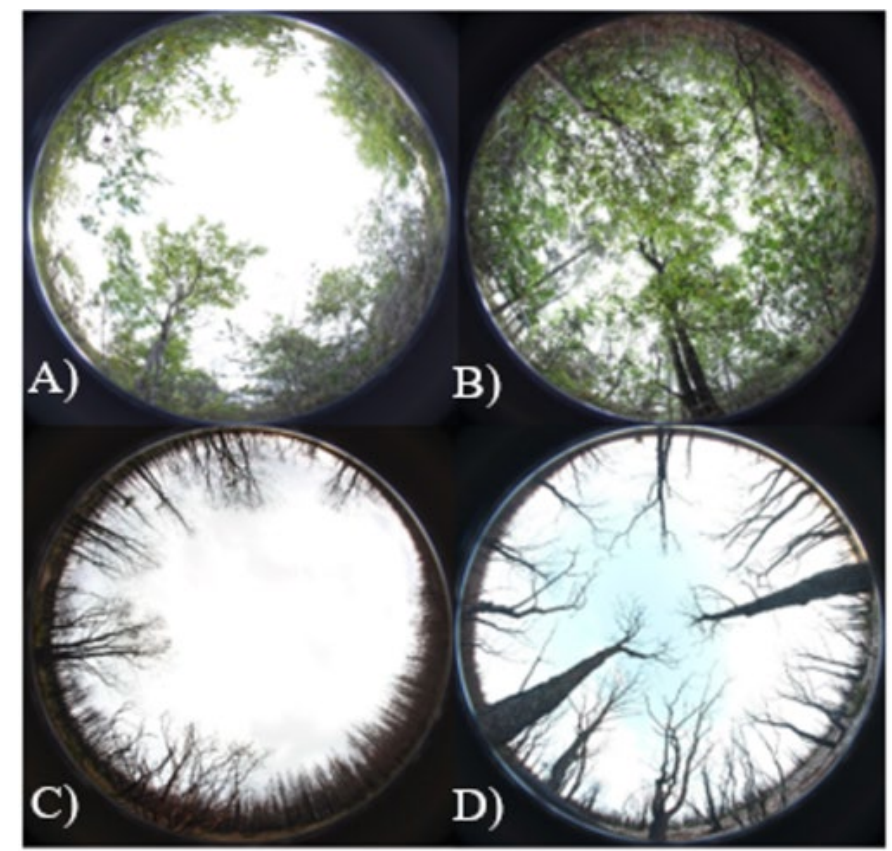

Figura 5. Fotografías hemisféricas del (A) matorral y (B) bosque preincendio y fotografías hemisféricas del (C) matorral y (D) bosque postincendio.

Figure 5. Hemispheric photographs of (A) scrub and (B) pre-fire forest and hemispheric photographs of $(C)$ scrub and (D) post-fire forest.

muy pequeña de DSF aportada por los denominados "sunflecks" o destellos de sol [11]. En general, la ISF, en doseles cerrados permite una mejor distribución de la radiación en los mismos [12]. Por el contrario, la DSF puede provocar efectos perjudiciales al dar pie a fenómenos de fotoinhibición en la vegetación [13], [14].

Por otra parte, los matorrales abiertos, como en este caso, permiten el paso de una gran fracción de la radiación. Este tipo de doseles son muy favorables para el desarrollo de la vegetación ya que elimina la radiación perjudicial (DSF) sin comprometer el proceso de fotosíntesis en las plantas bajo dosel [15], [16].

Valladares [17] señala que, en general los matorrales, en comparación con los bosques, permiten el paso de mayor radiación a través del dosel, coincidiendo así, los resultados obtenidos en este estudio, con los indicados por este autor.

Por otra parte, se encentra el LAI, el promedio para el matorral fue de $0,54 \pm 0,29$ y para el bosque fue de 1,68 $\pm 0,20$ (figura 7); más del triple que para el matorral.

Si se tiene en cuenta que, un LAl cercano o igual a 2 indica que el dosel es muy denso [18], entonces se corrobora que la estructura del dosel del bosque era mucho más compleja que la estructura del matorral, en lo que se refiere a la radiación incidente entre la atmósfera y el suelo bajo el dosel, y a los procesos ecofisiológicos de las plantas en esta cobertura [19], [20].

En cuanto al GndCover existía, antes del incendio, una brecha bastante amplia entre ambas coberturas, ya que el dosel del bosque cubría el 0,58 $\pm 0,09$ del suelo mientras que el matorral solo abarcaba el 0,00033 \pm 0,00017 (figura 8). En general los bosques tienden a mayores valores de índice de cobertura de suelo, en contraste con los matorrales [17].

Para la condición post-incendio, en las tres variables cuantificadas, GSF, LAl y GndCover, no se obtuvieron diferencias estadísticamente significativas, entre las coberturas de bosque y matorral, con $\mathrm{P} \leq 0,05$.

En cuanto al GSF se tiene que, para el bosque el promedio correspondió a $0,91 \pm 0,01$; por otra parte, para el matorral el promedio correspondió a 0,93 $\pm 0,04$ (figura 6). Si se tiene en cuenta que la radiación potencial total (GSF) óptima, para la supervivencia de la mayoría de las especies vegetales, se encuentra entre un 0,15 y un 0,40; es claro el panorama desalentador contra el cual se tendrá que enfrentar la nueva vegetación que se pueda establecer [17], [21], [22].

Que el valor de GSF en ambas coberturas sea tan alto puede traer consecuencias para la vegetación que pueda regenerarse, pues a pesar que la radiación representa un elemento fundamental para la realización de la fotosíntesis y demás procesos fisiológicos de la plantas, una intensidad luminosa que sobrepase el nivel de adaptación de las mismas, causa estrés, reduciéndose así la capacidad fotosintética, fenómeno conocido como fotoinhibición, además que muchos otros factores del microclima se ven afectados [19], [20]. La temperatura ambiental puede aumentar inclusive entre $2{ }^{\circ} \mathrm{C}$ y $3,5^{\circ} \mathrm{C}$, durante las horas de mayor radiación en el día, mientras que, durante la noche, los sitios incendiados pueden alcanzar temperaturas de hasta $1,4{ }^{\circ} \mathrm{C}$ menos con respecto a sitios no incendiados. La humedad relativa puede disminuir hasta en un $80 \%$ en comparación a los sitios no afectados por incendios forestales [23].

Por su parte, el LAl promedio para el bosque fue de $0,17 \pm 0,03$ y para el matorral de 0,21 $\pm 0,09$ (figura 7 ), los cuales son valores muy bajos para este índice [24], [25]. Estos resultados son similares a los obtenidos por Ruano, et al. [26], dichos autores determinaron que para bosques y matorrales mediterráneos, afectados por incendios forestales, el LAI no era mayor a 0,30; a escasos meses de ocurrido el incendio.

Si se tiene en cuenta que el LAl es un parámetro importante de la estructura del dosel; ya que cuantifica y caracteriza la interfaz activa entre la atmósfera y el suelo bajo la misma, la cual además, controla el tipo, 


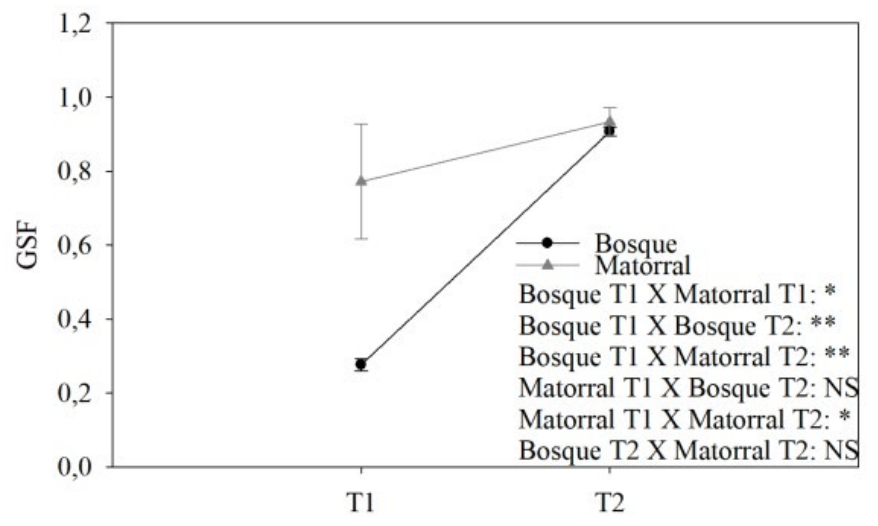

Figura 6. Factor global de sitio para ambas coberturas y para las dos condiciones, pre-incendio (T1) y post-incendio (T2). Donde *: significativo a $\mathrm{P} \leq 0,05 ;{ }^{* *}$ : significativo a $\mathrm{P} \leq 0,01$ y NS: sin diferencia estadísticamente significativa.

Figure 6. Global factor of site for both coverages and for the two conditions, pre-fire (T1) and post-fire (T2). Where *: significant at $P$ $\leq 0.05$; ${ }^{* *}$ : significant at $\mathrm{P} \leq 0.01$ and NS: no statistically significant difference.

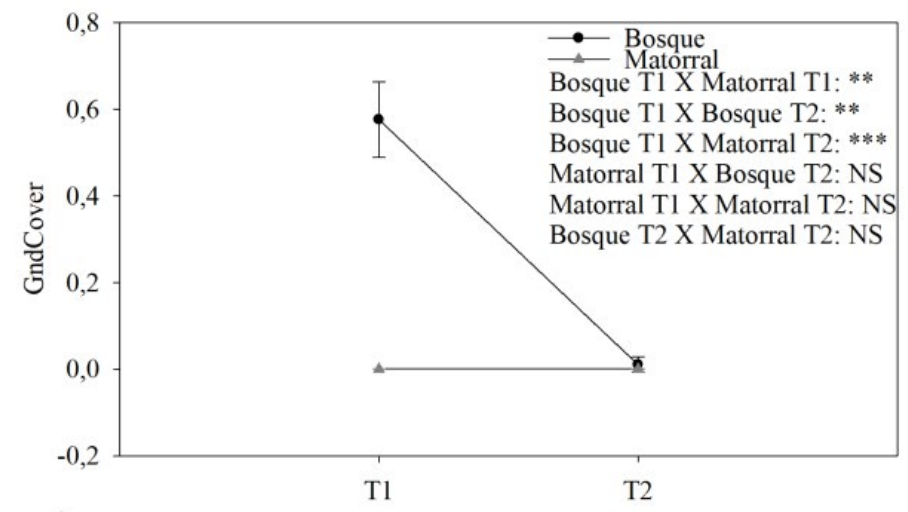

Figura 8. Índice de cobertura de suelo para ambas coberturas y para las dos condiciones, pre-incendio (T1) y post-incendio (T2). Donde ${ }^{* *}$ : significativo a $\mathrm{P} \leq 0,01 ;{ }^{* * *}$ : significativo a $\mathrm{P} \leq 0,001$ y NS: sin diferencia estadísticamente significativa.

Figure 8. Soil coverage index for both coverages and for the two conditions, pre-fire (T1) and post-fire (T2). Where ${ }^{* *}$ : significant at $\mathrm{P}$ $\leq 0.01 ;{ }^{* \star *}$ : significant at $\mathrm{P} \leq 0.001$ and NS: no statistically significant difference.

cantidad, calidad, distribución espacial y en el tiempo de la radiación incidente; entonces el que ambas coberturas tengan valores de este índice tan bajos, resulta realmente perjudicial, y más aún si se agrega que los valores de LAl publicados, para varios de los ecosistemas a nivel mundial, oscilan entre 0,40 y 16,9 [24], [25].

Lo anterior tiene graves repercusiones, debido a que el follaje es de vital importancia para muchos procesos fisiológicos en las plantas, como la fotosíntesis, el

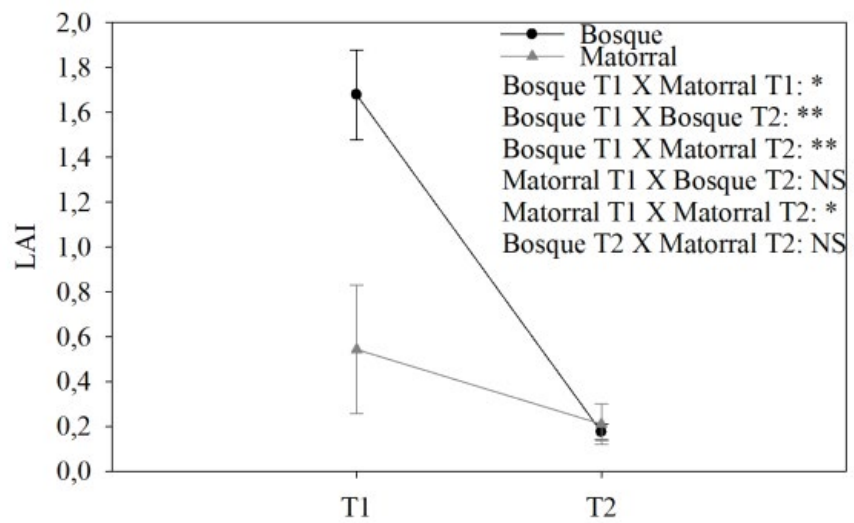

Figura 7. Índice de área foliar para ambas coberturas y para las dos condiciones, pre-incendio (T1) y post-incendio (T2). Donde *: significativo a $\mathrm{P} \leq 0,05$; **: significativo a $\mathrm{P} \leq 0,01$ y NS: sin diferencia estadísticamente significativa.

Figure 7. Leaf area index for both coverages and for the two conditions, pre-fire (T1) and post-fire (T2). Where *: significant at $\mathrm{P} \leq 0.05$; ${ }^{*}$ : significant at $\mathrm{P} \leq 0.01$ and NS: no statistically significant difference.

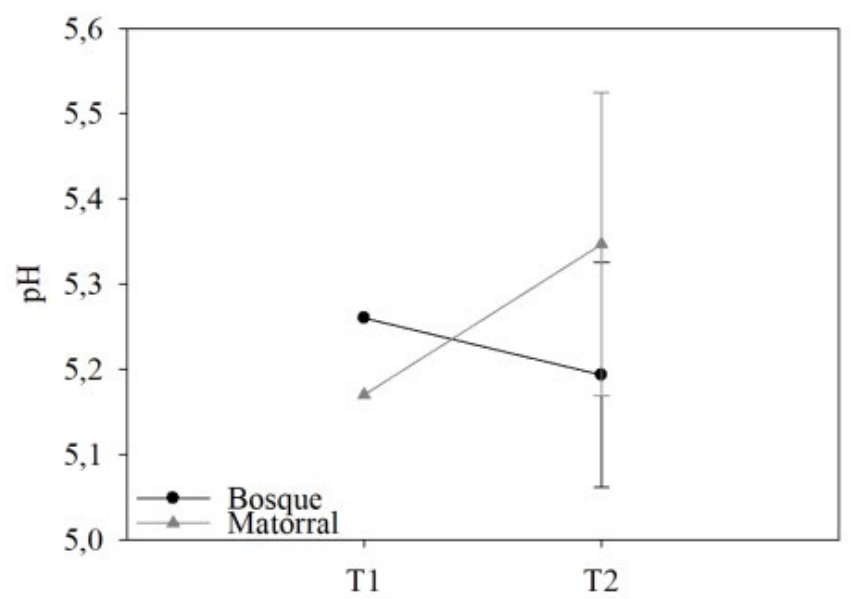

Figura 9. pH para ambas coberturas y para dos condiciones, preincendio (T1) y post-incendio (T2).

Figure 9. $\mathrm{pH}$ for both coverages and for two conditions, pre-fire (T1) and post-fire (T2).

intercambio gaseoso, y el crecimiento, además de su función de nodriza para la nueva vegetación que se establece bajo dosel [27].

Por otra parte, para el GndCover, aunque el promedio para el bosque fue de 0,01 $\pm 0,02$ y para el matorral de 0,00013 $\pm 0,00006$ (figura 8); no existe diferencia estadísticamente significativa, con $\mathrm{P} \leq 0,05$; debido a la gran variabilidad, en cuanto este índice, tanto en la cobertura de bosque, como en el matorral, además 


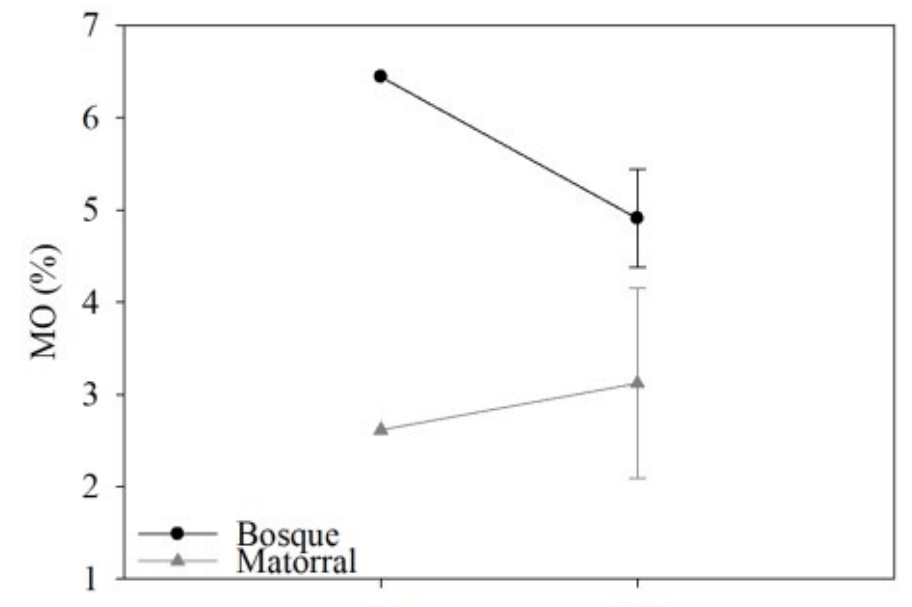

$\mathrm{Tl}$

$\mathrm{T} 2$

Figura 10. Materia orgánica para ambas coberturas y para las dos condiciones, pre-incendio (T1) y post-incendio (T2)..

Figure 10. Organic material for both coverages and for the two conditions, pre-fire (T1) and post-fire (T2).

corresponden a valores muy bajos, demostrando así la susceptibilidad a fenómenos de erosión, ya sea hídrica o eólica, lixiviación de nutrientes, pérdida del banco de semillas, entre otros [28].

Se puede inferir que, los efectos de la temporada de incendios forestales 2016-2017 en el dosel del bosque y matorral, específicamente cuantificados en los índices GSF, LAl y GndCover muestran un cambio drástico de la condición pre-incendio a post-incendio (figura 5).

Es así como, la comparación por condición y cobertura, para el GSF, indica que el incendio tuvo un efecto drástico, si se tiene que es lógico que las comparaciones Bosque T1 X Bosque T2, Bosque T1 X Matorral T2, y Matorral T1 $X$ Matorral T2, sean estadísticamente distintos (figura 6).

La diferencia estadísticamente significativa, en la comparación Bosque T1 X Matorral T1 (figura 6), hace referencia a que uno de los dos doseles evaluados era mucho más complejo y denso, en este caso el del bosque.

El que las comparaciones Matorral T1 $\mathrm{X}$ Bosque T2 y Bosque T2 $X$ Matorral T2 no registraran diferencia estadísticamente significativa (figura 6), demuestra la pérdida de complejidad y densidad del dosel del bosque.

Para el LAI se registró el mismo resultado que para el GSF, en las comparaciones (figura 7).

En cuanto al GndCover se obtuvo prácticamente el mismo resultado, excepto en la comparación de Matorral T1 X Matorral T2, los cuales no son estadísticamente distintos (figura 8).

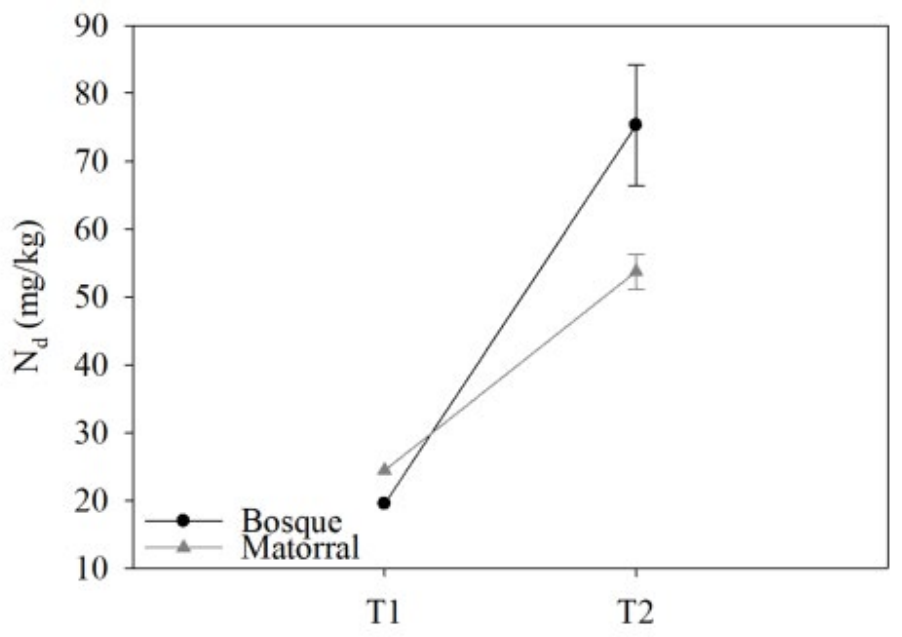

Figura 11. Nitrógeno disponible para ambas coberturas y para las dos condiciones, pre-incendio (T1) y post-incendio (T2).

Figure 11. Nitrogen available for both coverages and for the two conditions, pre-fire (T1) and post-fire (T2).

\section{Variables químicas del suelo, para ambas condiciones y coberturas}

En el caso del pH los valores pre y post-incendio, para los suelos de ambas coberturas, se encuentran en un rango similar, así para el suelo del bosque el valor preincendio era de 5,26 y su valor post-incendio de 5,19 \pm 0,13 . Por otra parte para el suelo del matorral el valor pre-incendio era de 5,17 ; mientras que su valor postincendio corresponde a 5,35 $\pm 0,18$ (figura 9).

Los efectos de los incendios sobre las propiedades químicas del suelo dependen de múltiples factores, como por ejemplo la intensidad, temperaturas alcanzadas, duración del evento, biomasa disponible, pendiente, vegetación, entre otros, obteniéndose así diferentes comportamientos y resultados entre diferentes suelos afectados por incendios forestales, e inclusive dentro de un mismo suelo [29], [58].

A pesar de que múltiples autores como Kutiel, et al. [30]; González, et al. [31]; Ulery, et al. [32] y Ulery, et al. [33] han demostrado que el $\mathrm{pH}$, generalmente tiende al aumento, después de un incendio, en el caso de este estudio no fue así para la cobertura de bosque, y en el caso del matorral, el ligero aumento percibido podría deberse más bien a la variabilidad a causa del análisis y no tanto a los efectos del incendio.

Por otra parte, también se ha demostrado que los aumentos de $\mathrm{pH}$ suelen ser efímeros [34-37] es decir, se da un aumento significativo durante las primeras semanas después del incendio, para posteriormente decrecer a causa de los efectos de la erosión eólica, 


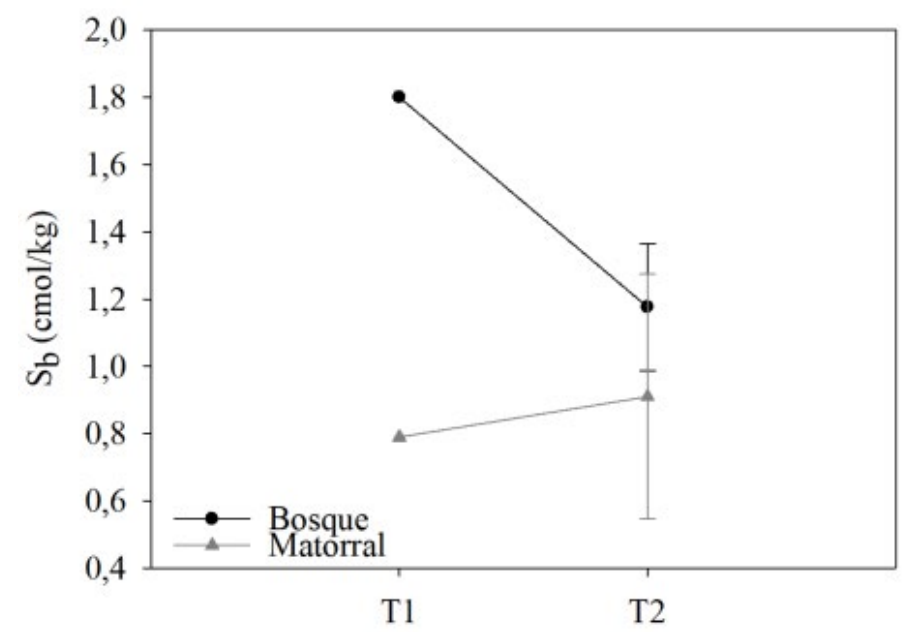

Figura 12. Suma de bases para ambas coberturas y para las dos condiciones, pre-incendio (T1) y post-incendio (T2).

Figure 12. Sum of bases for both coverages and for the two conditions, pre-fire (T1) and post-fire (T2).

además del lavado y arrastre de cationes por las lluvias, por lo que a los incrementos iniciales de $\mathrm{pH}$ tras el fuego, pueden seguir fuertes descensos que logran alcanzar valores similares e inclusive inferiores a los registrados antes del incendio [38], [39].

Esto podría explicar los niveles de $\mathrm{pH}$ tan similares entre las dos condiciones y para ambas coberturas, ya que en la comuna de Constitución, y en la Región del Maule en general, las precipitaciones se concentran en los meses de mayo a septiembre [65], si se tiene en cuenta que el incendio fue controlado el 10 de febrero del 2017 y las muestras post-incendio se colectaron el 3 de agosto, justo coincide con el periodo de precipitaciones que pudieron dar origen al lavado de ceniza y a la disminución del $\mathrm{pH}$ hasta niveles similares pre-incendio para ambas coberturas.
Es importante mencionar que los niveles de $\mathrm{pH}$ actuales podrían afectar la disponibilidad de nutrientes para la vegetación que se pueda regenerar, si se tiene en cuenta que el pH óptimo se encuentran entre 6,5 y 8 ; además que sigue existiendo el riesgo de que los valores para esta variable sigan disminuyendo en los suelos de ambas coberturas [38].

En cuanto a la $\mathrm{MO}$, en la cobertura de bosque se observó un descenso de 6,44 a 4,91 $\pm 0,53 \%$, mientras para el matorral se notó un ligero aumento de 2,62 a 3,12 $\pm 1,03 \%$ (figura 10).

Esta reducción de la $\mathrm{MO}$ orgánica en la cobertura de bosque y aumento en el matorral hace pensar que el incendio tuvo mayor severidad e intensidad en el bosque que en el matorral, posiblemente debido a diferencias en la carga de combustibles entre las coberturas. Cuesta \& Giraldo [38], De las Heras, et al. [39], y Mataix-Solera, et al. [40] indican que en incendios de menor intensidad puede haber incrementos de materia orgánica, por otra parte, a intensidades elevadas la cantidad de materia orgánica de la superficie del suelo tiende al descenso.

Por otra parte, se debe tener en cuenta que esta reducción de $\mathrm{MO}$ en el suelo del bosque puede traer consecuencias en la fertilidad, ya que la reducción en los contenidos de $\mathrm{MO}$ se traduce en un descenso de la capacidad de intercambio catiónico $(\mathrm{CIC})$ en proporción casi directa a dicha reducción [41].

En fuegos de menor intensidad, el cual parece el caso de la cobertura del matorral, los cationes son retenidos por el complejo absorbente, al no haberse visto afectada negativamente la $\mathrm{MO}$, pudiendo aumentarse así la fertilidad del suelo [42].

Para el nitrógeno disponible (Nd), en ambas coberturas, se dio un aumento marcado, de la condición pre a postincendio. En el caso del bosque de 19,50 a 75,30 $\pm 8,85$ $\mathrm{mg} / \mathrm{kg}$, por otra parte, en el matorral se pasó de 24,43 a $53,70 \pm 2,63 \mathrm{mg} / \mathrm{kg}$ (figura 11).

Cuadro 1. Aporte, en $\mathrm{cmol} / \mathrm{kg}$, de cada una de las bases intercambiables, al total de la suma.

Table 1. Contribution, in $\mathrm{cmol} / \mathrm{kg}$, of each of the interchangeable bases, to the total of the sum.

\begin{tabular}{|ccccc|}
\hline & bosque $(\mathrm{T} 1)$ & matorral $(\mathrm{T} 1)$ & bosque (T2) & matorral (T2) \\
\hline$K_{i}$ & 0,26 & 0,09 & $0,22 \pm 0,02$ & $0,14 \pm 0,04$ \\
$\mathrm{Ca}_{\mathrm{i}}$ & 1,04 & 0,48 & $0,60 \pm 0,11$ & $0,46 \pm 0,26$ \\
$\mathrm{Mg}_{\mathrm{i}}$ & 0,44 & 0,19 & $0,33 \pm 0,07$ & $0,29 \pm 0,09$ \\
$\mathrm{Na}_{\mathrm{i}}$ & 0,06 & 0,03 & $0,03 \pm 0,02$ & $0,02 \pm 0,01$ \\
\hline $\mathrm{S}_{\mathrm{b}}$ & 1,80 & 0,79 & $1,18 \pm 0,19$ & $0,91 \pm 0,36$ \\
\hline
\end{tabular}

${ }^{*}$ T1: Pre-incendio y T2: Post-incendio *T1: Pre-fire y T2: Post-fire

* T1: Pre-fire and T2: Post-fire * T1: Pre-fire and T2: Post-fire 


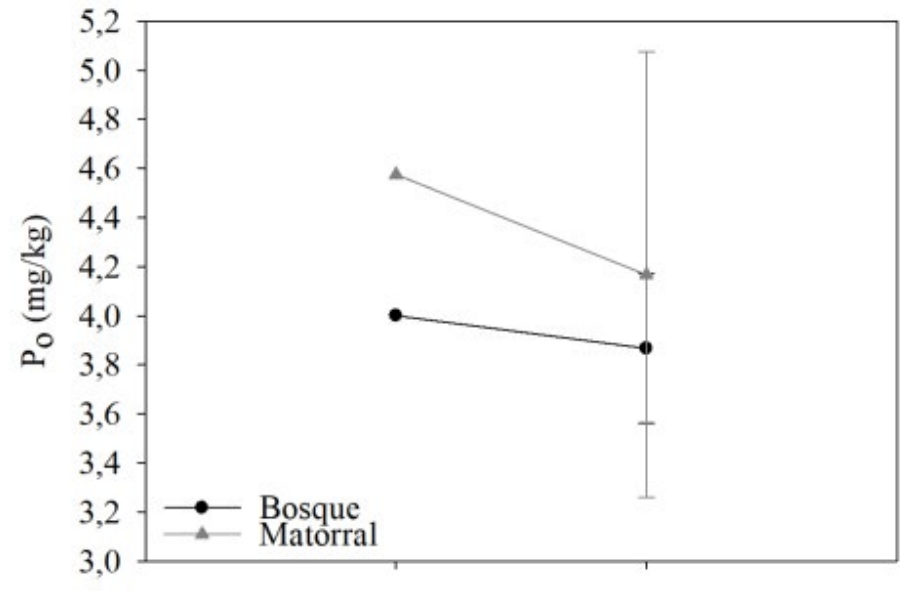

$\mathrm{T} 1$

$\mathrm{T} 2$

Figura 13. Fósforo de Olsen para ambas coberturas y para las dos condiciones, pre-incendio (T1) y post-incendio (T2).

Figure 13. Olsen phosphorus for both coverages and for the two conditions, pre-fire (T1) and post-fire (T2).

El incremento del nitrógeno disponible después de un incendio forestal suele ser mayor en los suelos más afectados, así el mayor aumento del $\mathrm{Nd}$ en el suelo del bosque, podría indicar, nuevamente, lo que antes se dedujo de la $\mathrm{MO}$, que el fuego fue, muy probablemente, más intenso en el bosque que en el matorral [31].

Parece ser una tendencia el hecho del aumento del Nd [36], [43-45], después de un incendio forestal; esto puede explicarse pues a pesar que el $\mathrm{Nd}$ es susceptible a ser volatilizado a temperaturas cercanas a los $200{ }^{\circ} \mathrm{C}$, esto no sucede ya que a esta temperatura ya se han iniciado los procesos de degradación de compuestos orgánicos de la vegetación, de la materia orgánica del suelo, de los microorganismos, etc., y la liberación desde complejos minerales del suelo, en los que previamente el $\mathrm{Nd}$ era inaccesible [36], [43], [46], por tanto no son extraños los aumentos en los niveles de $\mathrm{Nd}$, debido a la gran cantidad que es susceptible de ser mineralizado por efecto del fuego [44] .

En el caso de la suma de bases (Sb), fósforo de Olsen (Po) y potasio disponible (Kd), el comportamiento para ambas coberturas, tras el paso del incendio, fue contrario, excepto en el Po donde, tanto el suelo del bosque como el suelo del matorral, experimentaron decrecimiento.

Así para la Sb, el bosque pasó de 1,80 a 1,18 \pm 0,19 cmol/ $\mathrm{kg}$, mientras que en el matorral se dio un ligero aumento de 0,79 a $0,91 \pm 0,36 \mathrm{cmol} / \mathrm{kg}$ (figura 12).

Como consecuencia de la combustión de la vegetación y de la hojarasca, se liberan nutrientes que estaban

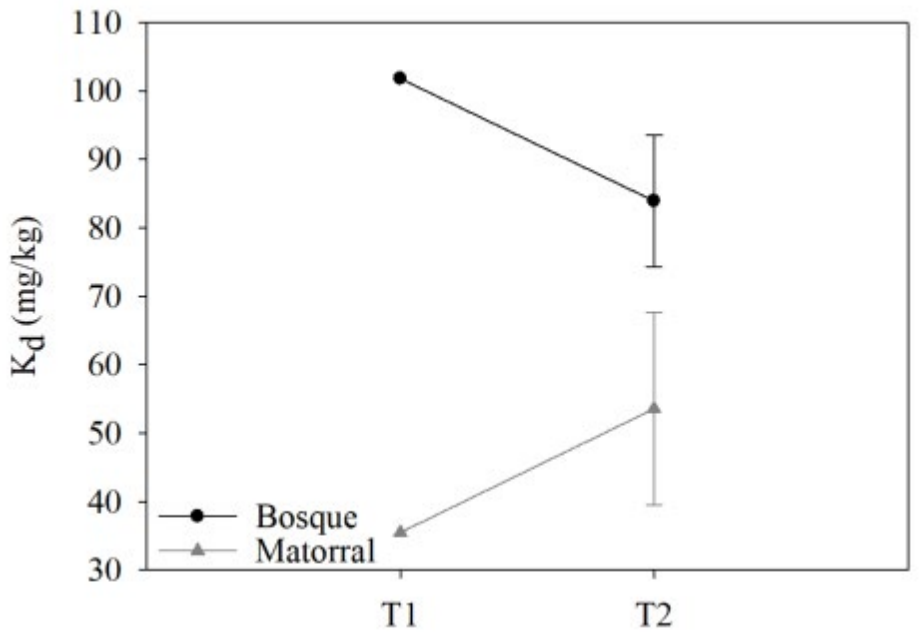

Figura 14. Fósforo de Olsen para ambas coberturas y para las dos condiciones, pre-incendio (T1) y post-incendio (T2).

Figure 14. Olsen phosphorus for both coverages and for the two conditions, pre-fire (T1) and post-fire (T2).

inmovilizados en ellas [47]. Así, nutrientes como el Ca $y$ el $\mathrm{K}$ pueden ser devueltos por las cenizas [48], sin embargo, no sucedió así en el suelo de la cobertura de bosque en donde se notó un descenso en los niveles de las cuatro bases intercambiables siendo este descenso más marcado en el Cai y el Mgi. Por el contrario, en el matorral se notó un aumento en los niveles de Ki y Mgi.

Este descenso en la fertilidad en el suelo del bosque y aumento de la misma en el suelo del matorral, reflejan de nuevo los efectos de la MO ya que en el bosque se redujeron los niveles de esta, reduciéndose también la CIC y con ella la capacidad de retener nutrimentos en el suelo, por su parte en el matorral al aumentarse los niveles de $\mathrm{MO}$ se favoreció el CIC y, probablemente debido a eso, se puede notar un aumento en los niveles de Ki y Mgi y por ende de la Sb [41].

En cuanto al Po, el suelo del bosque pasó de 4,00 a $3,87 \pm 0,31 \mathrm{mg} / \mathrm{kg}$, por otra parte, en el matorral el valor para esta variable decreció de 4,58 a 4,17 $\pm 0,91 \mathrm{mg} / \mathrm{kg}$ (figura 13).

El descenso de los niveles de Po, en el suelo de ambas coberturas, parece estar atribuido más bien a la variabilidad del análisis, debido a la magnitud del descenso, tanto en el bosque como en el matorral; en todo caso Soto, et al. [49] y Saa, et al. [50] apuntan la posibilidad de pérdida de $\mathrm{P}$ a la escorrentía y erosión post-fuego.

Por su parte, para el Kd, el suelo del bosque experimentó un decrecimiento de 17,90 mg/kg, al pasar de 101,80 a 
$83,90 \pm 9,64 \mathrm{mg} / \mathrm{kg}$, en el matorral, por el contrario, se dio un aumento de 35,48 a $53,57 \pm 14,05 \mathrm{mg} / \mathrm{kg}$ (figura 14).

El aumento de los niveles de $\mathrm{K}$ en el matorral, después del incendio, puede deberse a que este elemento es abundante en los tejidos vegetales, y como se cuantificó un incremento en los porcentajes de $\mathrm{MO}$ en esta cobertura, también es lógico que los niveles de $\mathrm{K}$ incrementen, lo que también explica la disminución de dicho elemento en el suelo del bosque [51].

\section{Diversidad y abundancia de especies arbóreas y arbustivas, para el bosque y matorral pre- incendio}

En el bosque, la densidad de individuos de especies arbóreas fue de 9642 individuos ha-1, de los cuales el $98 \%$ estaba representado por $N$. glauca. Por su parte para el matorral, la densidad de individuos de especies arbóreas, era de 697 individuos ha ${ }^{-1}$, en donde N. glauca representaba el $65 \%$, seguido de Luma apiculata ((DC.) Burret) con un $30 \%$ y Cryptocarya alba ((Molina) Looser) con un $2 \%$; es importante resaltar que en esta cobertura se encontraron dos especies que en el bosque no había, estas eran Kageneckia oblonga (Ruiz \& Pav.) y L. apiculata, por otra parte en el bosque se hallaban dos especies que en el matorral no se encontraron, estas fueron Lithraea caustica ((Molina) Hook. \& Arn.) y Lomatia dentata ((Ruiz \& Pav.) R. Br.)
Para las especies arbustivas, en el bosque, existía una densidad de 2056 individuos ha-1 ${ }^{-1}$, de los cuales el $64 \%$ correspondía a Azara integrifolia (Ruiz \& Pav.), un $12 \%$ lo constituía Ugni molinae (Turcz.), un $10 \%$ Aristotelia chilensis ((Molina) Stuntz) y Escallonia pulverulenta ((Ruiz \& Pav.) Pers.) junto con Baccharis rhomboidalis (J.Rémy) un $7 \%$ cada una.

Para la condición de matorral, la densidad de especies arbustivas era ligeramente superior a la cobertura de bosque, con 2304 individuos ha ${ }^{-1}$, además la proporción estaba mejor distribuida, en donde $E$. pulverulenta representaba un $30 \%, A$. integrifolia un $27 \%$; $B$. rhomboidalis un $18 \%$, Gochnatia foliolosa ((D. Don) Cabrera) un $16 \%$, U. molinae un $5 \%$, A. chilensis un $3 \%$ y Rosa moschata (Mill.) con un $1 \%$. Además, para esta cobertura se econtró mayor diversidad de especies arbustivas ya que ni $G$. foliolosa ni $R$. moschata se encontraron en la cobertura de bosque (figura 15).

\section{Diversidad y abundancia de especies arbóreas y arbustivas, para el bosque y matorral post- incendio}

Ocho meses después del incendio, del total de especies arbóreas registradas en la condición pre-incendio, sólo $N$. glauca presentaba regeneración natural, por medio de rebrotes, principalmente en la base de los árboles sobrevivientes, con 561 individuos ha-1 en el bosque y 34

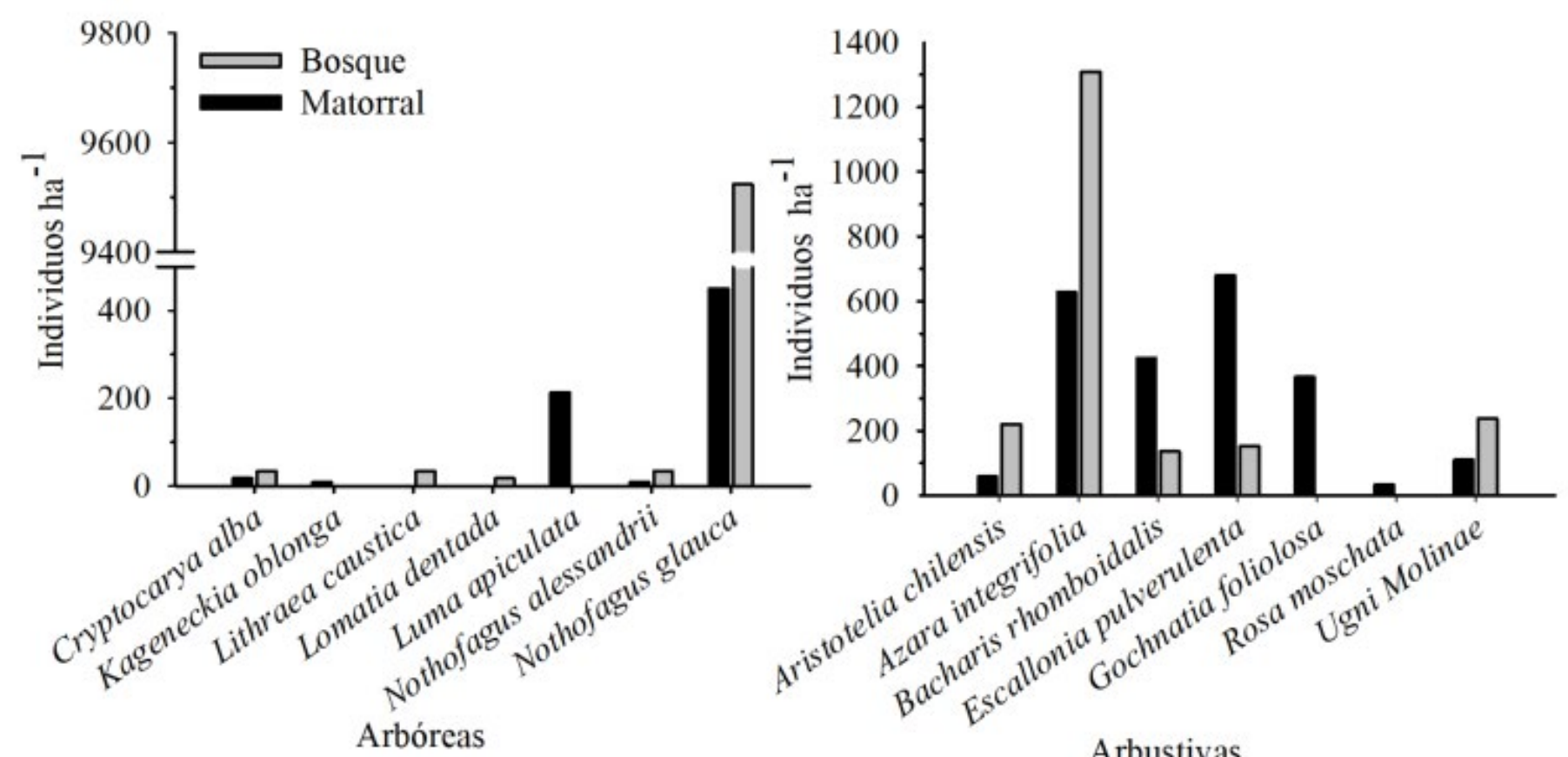

Figura 15. Densidad de individuos por hectárea de especies arbóreas y arbustivas para el bosque y matorral pre-incendio.

Figure 15.Density of individuals per hectare of arboreal and shrub species for the forest and pre-fire scrub. 
individuos ha-1 en el matorral (figura 16). En cuanto a las especies arbustivas no se encontró regeneración, tanto en el bosque como en el matorral (figura 16).

Si bien es cierto, no se puede emitir un criterio definitivo, a ocho meses de ocurrido el incendio, en cuanto a la degradación y pérdida de diversidad y abundancia de especies arbóreas y arbustivas, tanto para el bosque como para el matorral, ya que se ha documentado, para ecosistemas nativos de la zona central de Chile, que inclusive después de periodos de treinta años, los efectos de los incendios aún persisten [52], en este sentido la alteración en la estructura de la vegetación se manifiesta evidentemente en una escala de mucho mayor tiempo [53].

No obstante si se comparan los resultados obtenidos con los publicados por Quintanilla [54], el cual indica que para especies como $L$. caustica se han encontrado rebrotes a partir de lignotuber aproximadamente a los 45 días de generado el incendio, otra especie arbórea con una relativa rápida regeneración corresponde a C. alba, para el género Baccharis se ha demostrado una mayor capacidad de recuperación y colonización, inclusive un año después de los incendios, se puede notar que el sitio de estudio presenta un panorama complicado.
El hecho que no se encontrara regeneración para las especies arbustivas es un resultado que concuerda con lo documentado por Verzino, et al. [52], los cuales mencionan que la riqueza de especies arbustivas es menor en ecosistemas incendiados recientemente, debido a que algunas especies arbustivas más delicadas son destruidas totalmente por el fuego y tardan periodos de años en reinstalarse.

Lo anterior hace referencia a lo que múltiples autores como Blackhall, et al. [23], Verzino, et al. [52], Nasi, et al. [55], Castillo, et al. [56], y Gallegos, et al. [57], mencionan, que en bosques que no están adaptados al fuego, como es el caso del tipo forestal Roble-Hualo, éste causa graves consecuencias obstaculizando así la recuperación de las especies originales, resultando en una pérdida importante de especies, cambios en la estructura y composición florística y en la capacidad de sustento del sistema natural. Es así como los incendios son responsables de cambios sustanciales en la composición florística y en la fisionomía del paisaje.

\section{Conclusiones}

El incendio tuvo graves repercusiones sobre los doseles de ambas coberturas, provocando la perdida de

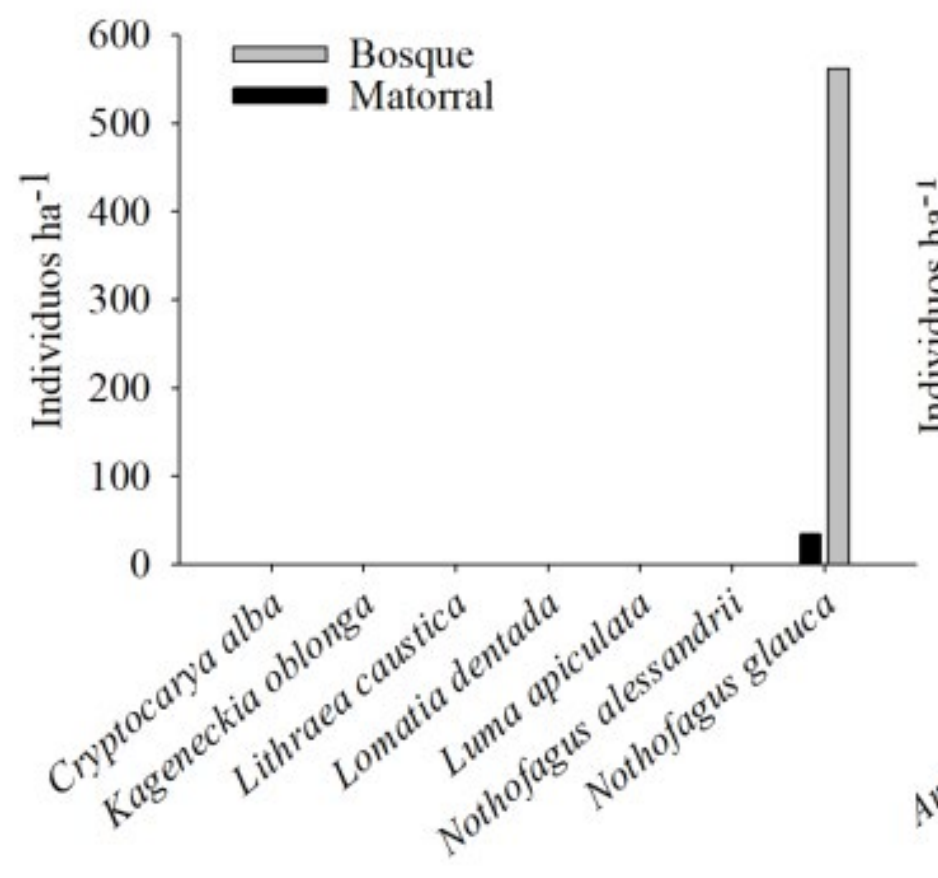

Arbóreas

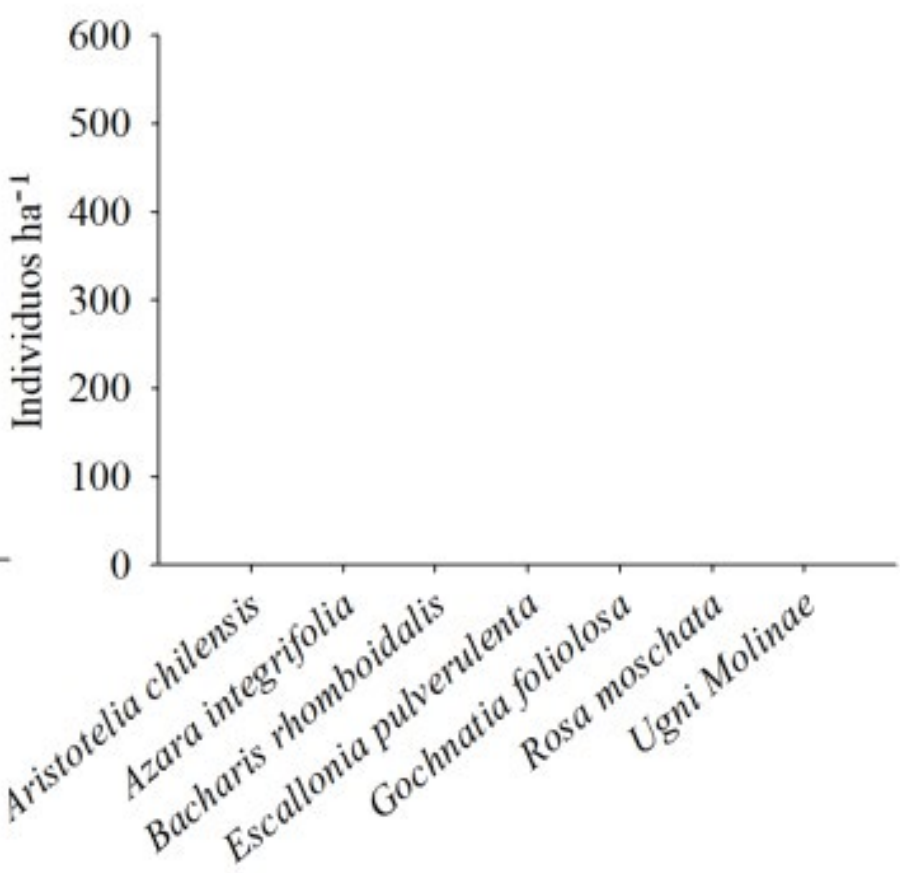

Arbustivas

Figura 16. Densidad de individuos por hectárea de especies arbóreas y arbustivas para el bosque y matorral post-incendio.

Figure 16. Density of individuals per hectare of arboreal and shrub species for the forest and post-fire scrub. 
densidad y complejidad de los mismos, resultando en aumento de la radiación potencial total, reducción del área foliar y de la cobertura del suelo, tanto en el matorral como en el bosque.

A seis meses después del incendio, el pH no es diferente de la condición pre-incendio, tanto en el suelo del matorral como en el del bosque, por otra parte en el suelo de esta última cobertura se observó un descenso en el porcentaje de $\mathrm{MO}$, al contrario del matorral; el $\mathrm{Nd}$ sufrió un considerable aumento después del incendio, en ambas coberturas, la $\mathrm{Sb}$ fue mayor en la condición post-incendio para el matorral, no así en el bosque, el mismo comportamiento se obtuvo para el $\mathrm{Kd}$, mientras que en el Po se dio un descenso en ambas coberturas de la condición pre a post-incendio.

Ocho meses después del incendio se encuentra escaza regeneración de N. glauca, tanto en el matorral como en el bosque, del resto de especies arbóreas y arbustivas no se registró regeneración natural, lo que indica una grave pérdida de diversidad y abundancia, y una depauperación de la composición del ecosistema del tipo forestal Roble-Hualo estudiado.

\section{Recomendaciones}

Debido a la gravedad de los efectos del incendio, sobre el sitio de estudio, se recomienda seguir las evaluaciones en el tiempo.

El uso de un sensor de radiación PAR en conjunto con el uso de fotografías hemisféricas, podría ser una herramienta muy útil para obtener una medida más exacta de la radiación bajo dosel.

Algunas técnicas de restauración podrían ser recomendables para la recuperación del sitio, por ejemplo, establecer medidas de conservación de suelo con el fin reducir los efectos de la erosión y lixiviación de nutrimentos, enmiendas de encalado para neutralizar el $\mathrm{pH}$, incorporación de $\mathrm{MO}$, esquemas de fertilización apropiados, control de combustibles en el suelo para frenar el avance y efectos negativos de posibles futuros incendios, enriquecimiento con especies pre-incendio, control de vegetación exótica invasora, puesto que el sitio de estudio está envuelto dentro de una matriz de plantaciones forestales de Pinus radiata D.Don.

\section{Agradecimientos}

Al Programa de Movilidad Estudiantil del Consejo Nacional de Rectores (CONARE), al Centro Tecnológico de la Planta Forestal del Instituto Forestal, sede Biobío.

\section{Referencias}

[[1] J. J. Armesto, C. Smith-Ramírez, M. R. Carmona, J. L. CelisDiez, I. A. Díaz, A. Gaxiola, et al., "Old-growth temperate rainforests of South America: conservation, plant-animal interactions, and baseline biogeochemical processes," in Old-growth forests, ed: Springer, 2009, pp. 367-390.

[2] S. R. Abella and E. Engel, "Influences of wildfires on organic carbon, total nitrogen, and other properties of desert soils," Soil Science Society of America Journal, vol. 77, pp. 18061817, 2013.

[3] I. Fernández, N. Morales, L. Olivares, J. Salvatierra, M. Gómez, and G. Montenegro, Restauración ecológica para ecosistemas nativos afectados por incendios forestales: Facultad de Agronomía e Ingeniería Forestal, Dirección de Investigación y Postgrado, Dirección de Extensión, Pontificia Universidad Católica de Chile, 2010.

[4] G. Ávila, M. Aljaro, and B. Silva, "Observaciones en el estrato herbáceo del matorral después del fuego," in Anales del Museo de Historia Natural de Valparaíso (Chile), 1981, pp. 99-105.

[5] G. Montenegro, R. Ginocchio, A. Segura, J. Keely, and M. Gomez, "Fire regimes and vegetation responses in two Mediterranean-climate regions," Revista chilena de historia natural, vol. 77, pp. 455-464, 2004.

[6] I. Quiroz, E. García, M. González, and H. Soto, "Evaluación de los métodos de regeneración aplicados en el tipo forestal Roble-Hualo en la Región del Maule," Ciencia e Investigación Forestal, vol. 17, pp. 325-358, 2011.

[7] A. M. Venegas and C. N. Meynard, "Avifauna de un bosque de Roble-Hualo en Chile Central," Boletín Chileno de Ornitología, vol. 11, pp. 18-22, 2005.

[8] I. F. Piñeiro, Influencia de los incendios forestales sobre la materia orgánica edáfica: Universidade de Santiago de Compostela, 1997.

[9] J. Landsberg, "El fuego y los bosques: El fuego puede ser un buen criado o un mal amo," in Actas del XI Congreso Forestal Mundial. Turquía.[en línea] www. fao. org/montes/ foda/wforcong/PUBLI/PDFN1S_T6. PDF, 1997.

[10] N. Urzúa and F. Cáceres, "Incendios forestales: principales consecuencias económicas y ambientales en Chile," RIAT Revista Interamericana de Ambiente y Turismo, vol. 7, pp. 18-24, 2011.

[11] R. W. Pearcy, R. L. Chazdon, L. J. Gross, and K. A. Mott, "Photosynthetic utilization of sunflecks: a temporally patchy resource on a time scale of seconds to minutes," Exploitation of environmental heterogeneity by plants, pp. 175-208, 1994.

[12] L. Gu, D. Baldocchi, S. B. Verma, T. Black, T. Vesala, E. M. Falge, et al., "Advantages of diffuse radiation for terrestrial ecosystem productivity," Journal of Geophysical Research: Atmospheres, vol. 107, pp. 1-23, 2002.

[13] T. Marañón, J. J. Camarero, J. Castro, M. Díaz, J. M. Espelta, A. Hampe, et al., "Heterogeneidad ambiental y nicho de regeneración," Ecología del bosque mediterráneo en un mundo cambiante, pp. 69-99, 2004.

[14] M. Zavala, R. Zamora, F. Pulido, J. A. Blanco, J. B. 
Imbert, T. Marañón, et al., "Nuevas perspectivas en la conservación, restauración y gestión sostenible del bosque mediterráneo," Ecología del bosque mediterráneo en un mundo cambiante, pp. 509-530, 2004.

[15] F. Valladares, "Architecture, ecology and evolution of plant crowns," Handbook of functional plant ecology, pp. 121194, 1999.

[16] F. Valladares, "Luz y evolución vegetal-En el curso de la evolución unas plantas se han adaptado a una vida sometida a radiaciones extremas y otras han desarrollado," Investigación y Ciencia: Edición Española de Scientific American, pp. 73-79, 2001.

[17] F. Valladares, "La disponibilidad de luz bajo el dosel de los bosques y matorrales ibéricos estimada mediante fotografía hemisférica," Ecología, vol. 20, pp. 11-30, 2006.

[18] A. Kundela, "Leaf Area Index estimation and radiation interception measurements in Chinese subtropical forests: assessment of methods in heterogeneous topography," Master thesis. University of Zurich, Institute of Evolutionary Biology and Enviromental Studies, 2009.

[19] T. Kitzberger, E. Raffaele, K. Heinemann, and M. J. Mazzarino, "Effects of fire severity in a north Patagonian subalpine forest," Journal of Vegetation Science, vol. 16, pp. 5-12, 2005.

[20] J. Paritsis, E. Raffaele, and T. T. Veblen, "Vegetation disturbance by fire affects plant reproductive phenology in a shrubland community in northwestern Patagonia, Argentina," New Zealand Journal of Ecology, pp. 387-395, 2006.

[21] J. Gómez, F. Valladares, and C. Puerta-Piñero, "Differences between structural and functional environmental heterogeneity caused by seed dispersal," Functional Ecology, vol. 18, pp. 787-792, 2004.

[22] F. Valladares and B. Guzmán, "Canopy structure and spatial heterogeneity of understory light in an abandoned Holm oak woodland," Annals of Forest Science, vol. 63, pp. 749761, 2006.

[23] M. Blackhall, E. Raffaele, and T. T. Veblen, "Efectos combinados del fuego y el ganado en matorrales y bosques del noroeste patagónico," Ecología austral, vol. 25, pp. 1-10, 2015.

[24] V. Le Dantec, E. Dufrêne, and B. Saugier, "Interannual and spatial variation in maximum leaf area index of temperate deciduous stands," Forest Ecology and Management, vol. 134, pp. 71-81, 2000.

[25] D. P. Turner, S. A. Acker, J. E. Means, and S. L. Garman, "Assessing alternative allometric algorithms for estimating leaf area of Douglas-fir trees and stands," Forest Ecology and Management, vol. 126, pp. 61-76, 2000.

[26] A. J. Ruano, F. Pérez, and R. M. Llovería, "Niveles de LAl/ fPAR en superficies afectadas por incendios forestales en Aragón. Análisis mediante el producto MCD15A2 DE MODIS," Pirineos, vol. 171, p. 019, 2016.

[27] D. Arias, J. Calvo, and A. Dohrenbusch, "Calibration of LAl-2000 to estimate leaf area index (LAl) and assessment of its relationship with stand productivity in six native and introduced tree species in Costa Rica," Forest Ecology and Management, vol. 247, pp. 185-193, 2007.
[28] E. Benito, M. Varela, and M. Rodríguez-Alleres, "Efectos de los incendios forestales en la erosionabilidad de los suelos en Galicia," Cuadernos de Investigación Geográfica, vol. 40, 2014.

[29] X. Úbeda, "Efectos de los incendios forestales sobre los suelos," Problemática Ambiental de Suelos Mediterráneos. Universidad Internacional Menéndez Pelayo UIMP. Alicante, pp. 118-137, 2000.

[30] P. Kutiel, Z. Naveh, and H. Kutiel, "The effect of a wildfire on soil nutrients and vegetation in an Aleppo pine forest on Mount Carmel, Israel," Fire in ecosystem dynamics: Mediterranean and northern perspectives, pp. 85-94, 1990.

[31] J. González, M. Fernández, and G. Gimeno, "Efectos de los incendios forestales sobre el suelo," Suelo y Planta, vol. 2, pp. 71-79, 1992.

[32] A. Ulery, R. Graham, and C. Amrhein, "Wood-ash composition and soil $\mathrm{pH}$ following intense burning," Soil Science, vol. 156, pp. 358-364, 1993.

[33] A. Ulery, R. Graham, O. Chadwick, and H. Wood, "Decadescale changes of soil carbon, nitrogen and exchangeable cations under chaparral and pine," Geoderma, vol. 65, pp. 121-134, 1995.

[34] P. Viro, "Effects of forest fire on soil," Fire and ecosystems, pp. 7-45, 1974.

[35] P. Khanna and R. Raison, "Effect of fire intensity on solution chemistry of surface soil under a Eucalyptus pauciflora forest," Soil Research, vol. 24, pp. 423-434, 1986.

[36] R. d. Celis, A. Jordán López, and L. M. Martínez Zavala, "Efectos del fuego en las propiedades biológicas, físicas y químicas del suelo," Grandes incêndios florestais, erosão, degradação e medidas de recuperação dos solos., pp. 145-160, 2013.

[37] L. Etiegni and A. Campbell, "Physical and chemical characteristics of wood ash," Bioresource technology, vol. 37, pp. 173-178, 1991.

[38] J. R. Cuesta and I. O. Giraldo, "Efectos de los incendios forestales en las propiedades del suelo. Estado del arte," CUADERNO ACTIVA, pp. 59-67, 2013.

[39] J. De las Heras, J. Martínez, and J. Herranz, "Impacto ecológico de los incendios forestales," Revista de estudios albacetenses, vol. 29, pp. 105-117, 1991.

[40] J. Mataix-Solera, I. Gómez, J. Navarro-Pedreño, C. Guerrero, and R. Moral, "Soil organic matter and aggregates affected by wildfire in a Pinus halepensis forest in a Mediterranean environment," International Journal of Wildland Fire, vol. 11, pp. 107-114, 2002.

[41] T. Carballas, "Effects of fires on soil quality. Biochemical aspects. Proyect: Fire influence on orgánic matter evolution and $\mathrm{N}$ and $\mathrm{P}$ mobilization in forest soils," Management for soil reclamation. Instituto de Investigaciones Agrobiológicas de Galicia (CSIC). pp. 249-261, 1993.

[42] J. Ibáñez, M. Lobo, G. Almendros, and A. Polo, "Impacto del fuego sobre algunos ecosistemas edáficos de clima mediterráneo continental en la zona centro de España," Boletín de la Estación central de Ecología, vol. 24, pp. 27 42, 1983.

[43] E. A. Smithwick, M. G. Turner, M. C. Mack, and F. S. 
Chapin, "Postfire soil N cycling in northern conifer forests affected by severe, stand-replacing wildfires," Ecosystems, vol. 8, pp. 163-181, 2005.

[44] M. G. Turner, E. A. Smithwick, K. L. Metzger, D. B. Tinker, and W. H. Romme, "Inorganic nitrogen availability after severe stand-replacing fire in the Greater Yellowstone ecosystem," Proceedings of the National Academy of Sciences, vol. 104, pp. 4782-4789, 2007.

[45] R. E. Boerner, J. Huang, and S. C. Hart, "Impacts of Fire and Fire Surrogate treatments on forest soil properties: a meta-analytical approach," Ecological Applications, vol. 19, pp. 338-358, 2009.

[46] Y. Rivas, D. Huygens, H. Knicker, R. Godoy, F. Matus, and P. Boeckx, "Soil nitrogen dynamics three years after a severe Araucaria-Nothofagus forest fire," Austral Ecology, vol. 37, pp. 153-163, 2012.

[47] J. Mataix Solera, "Modificaciones físico-químicas en suelos afectados por un incendio forestal," Tesis de Licenciatura. Facultad de Ciencias. Universidad de Alicante, 1997.

[48] J. Mataix-Solera, C. Guerrero, I. Gómez, J. NavarroPedreño, J. Mataix, and R. Moral, "Modifications in N, P, $\mathrm{K}, \mathrm{Na}, \mathrm{Ca}, \mathrm{Mg}$ and organic matter contents in a forest soil affected by experimental fire," in 6th International Meeting on Soils with Mediterranean Type of Climate. Barcelona. Extended Abstracts: 774, 1999.

[49] B. Soto, E. Benito, R. Pérez, R. Basanta, and F. DíazFierros, "Alterations in surface runoff due to forest fires," in Abstracts of the International Conference on Soil Erosion and Degradation as a Consequence of Forest Fires, European Society for Soil Conservation, Barcelona, 1991, p. 29.

[50] A. Saa, M. Trasar-Cepeda, and T. Carballas, "Soil P status and phosphomonoesterase activity of recently burnt and unburnt soil following laboratory incubation," Soil Biology and Biochemistry, vol. 30, pp. 419-428, 1998.

[51] J. Canadell and I. Fontanillas, "Els efectes del foc sobre el sol i els nutrients de lecosistema," Quaderns dEcologia Aplicada, vol. 10, pp. 145-156, 1987.

[52] G. Verzino, J. Joseau, M. Dorado, E. Gellert, S. Rodríguez Reartes, and R. Nóbile, "Impacto de los incendios sobre la diversidad vegetal, Sierras de Córdoba, Argentina," Ecología Aplicada, vol. 4, pp. 25-34, 2005.

[53] M. Castillo, "El cambio del paisaje vegetal afectado por incendios en la Zona Mediterránea Costera de la V Región," Santiago: Tesis Magíster Geografía. Universidad de Chile, 2006.

[54] V. Quintanilla, "Influencia del fuego en el desequilibrio ecológico de la vegetación en la zona mediterránea de Chile: casos de estudio," Investigaciones Geográficas, pp. Pág. 3-15, 2000.

[55] R. Nasi, R. Dennis, E. Meijaard, G. Applegate, and P. Moore, "Los incendios forestales y la diversidad biológica," Unasylva (FAO), 2002.

[56] M. Castillo, R. Garfias, G. Julio, and L. González, "Análisis de grandes incendios forestales en la vegetación nativa de Chile," Interciencia, vol. 37, 2012.

[57] A. Gallegos Rodríguez, G. A. González Cueva, R. Gerardo
Cabrera Orozco, C. Marcelli Sánchez, and E. Hernández Álvarez, "Efecto de la recurrencia de incendios forestales en la diversidad arbórea," Revista mexicana de ciencias forestales, vol. 5, pp. 110-125, 2014.

[58] Corporación Nacional Forestal CONAF, "Analisis de la afectación y severeidad de los incendios forestales ocurridos en enero y febrero de 2017 sobre los usos de suelo y los ecosistemas naturales presentes entre las regiones de Coquimbo y Los Ríos de Chile", Santiago, Chile, informe técnico, 2017.

[59] Infraestructura de Datos Geoespaciales-Chile, "Descarga de capas", Ministerio de Bienes Nacionales, 2017. [En línea]. Disponible en http://www.ide.cl/descarga/capas.html

[60] C. Albers, "Coberturas SIG para la enseñanza de la Geografía en Chile", Universidad de La Frontera, Temuco, Chile, 2012. [En línea]. Disponible en www.rulamahue.cl/mapoteca.

[61] M. Acevedo, "Técnicas silvícolas de establecimiento para Nothofagus glauca en bosques alterados del Tipo Forestal Roble-Hualo en la Cordillera de la Costa", Concepción, Chile, Informe Núm: 006/2015, 2017.

[62] N. Webb, "HemiView Manual Revision Number: 2.1.", University of Kansas Information Technoly and Telecommunication Centre (ITTC), the Kansas Technology Enterprise Corporation (KTEC), and the Kansas Biological Survey, Kansas, USA, 1999.

[63] R. A. Sadzawka, M. A. Carrasco, R. Grez, M.L. Mora, H. Flores, y A. Neaman. "Métodos de análisis de recomendados para los suelos de Chile", Instituto de Investigaciones Agropecuarias, Serie Actas INIA N 34, Santiago, Chile, 164 p, 2006.

[64] R. A. Sadzawka, M .A. Carrasco, R. Demanet, H. Flores, R. Grez, M.L. Mora, y A. Neaman, "Métodos de análisis de tejidos vegetales", Segunda Edición, Instituto de Investigaciones Agropecuarias, Serie Actas INIA N $\mathrm{N}^{\circ} 40$, Santiago, Chile, 140 p, 2007.

[65] DMC, "Climatología Regional", 2001. [En línea]. Disponible en http://164.77.222.61/climatologia/publicaciones/ Climatologia_regional.pdf

\section{Este artículo debe citarse como:}

Rosales-Rodríguez, J., Esquivel-Segura, E., AcevedoTapia, M., González-Ortega, M., \& Cartes-Rodríguez, E. (2018). Situación pre y post-incendio, de un ecosistema del tipo forestal Roble-Hualo, Región del Maule, Chile. Revista Forestal Mesoamericana Kurú, 16(38), 55-68. Doi. 10.18845/rfmk.v16i38.3997 